\title{
Unilateral panuveitis and retinal detachment: A rare complication of typhoid fever
}

\section{Dear Editor,}

The ocular manifestations in typhoid fever described include catarrhal conjunctivitis, ulcerative keratitis, keratomalacia, iridocyclitis, vitritis, optic neuritis, optic atrophy, choroiditis, paresis of accommodation, ptosis and abducens nerve palsy. ${ }^{1}$ The manifestations may set in acute stage of the disease mostly during the third week. ${ }^{1}$

Case report. A 57-year-old Asian Indian woman presented to us with blurring of vision in her left eye (LE) of 4 months duration following fever. The cause of the fever was not established. She had undergone one intraocular injection of anti-vascular endothelial growth factor (VEGF) elsewhere in her LE, 1 month prior to presentation to us with no appreciable improvement. Tests done elsewhere showed increased erythrocyte sedimentation rate: $40 \mathrm{~mm} / \mathrm{hr}$, negative C-reactive protein (CRP) and normal random blood sugar $(72 \mathrm{mg} / \mathrm{dL})$. Her visual acuity in the right eye (RE) was 20/20 and in the LE counting fingers close to the face. Anterior segment examination of the RE was normal and the LE cornea had nongranulomatous keratic precipitates, anterior chamber flare $2+$, cells $1+$ with complicated cataract (a type of cataract that occurs secondary to conditions like uveitis, retinitis pigmentosa, topical or systemic steroids) and total posterior synechiae with vitreous haze $2+$ and cells $1+$. Posterior segment examination of the LE showed retinitis, sub-retinal fluid, retinal detachment with retinal folds inferiorly with no rhegma or hole and the RE was normal (Fig. 1A). A diagnosis of panuveitis with combined exudative and tractional retinal detachment in the LE was made.

Optical coherence tomography (OCT) of the LE showed vitreo-macular traction, neurosensory detachment, distortion of retinal layers, retinal schisis, and disruption of photoreceptor layer involving fovea (Fig. 2). Our investigations showed erythrocyte sedimentation rate of $60 \mathrm{~mm} / \mathrm{hr}$; CRP of $16 \mathrm{mg} / \mathrm{L}$; Widal test O\&H (1:160 dilution) was positive; IgM for typhoid (by immunochromatography) was positive, suggestive of acute phase of typhoid; and dengue and chikungunya $\operatorname{IgM} / \operatorname{IgG}$ and Weil-Felix test were negative. Other autoimmune workup was negative. Mantoux was negative and chest X-ray was within normal limits. Blood culture for typhoid bacilli was not done. The patient was now started on oral ciprofloxacin 500mg twice daily and oral steroids after the physician clearance. Oral prednisolone $35 \mathrm{mg}$ (anti-inflammatory dose $0.5 \mathrm{mg} / \mathrm{kg}$ of body weight/ day) in tapering dose of $5 \mathrm{mg}$ every week was given for about 6 weeks along with ciprofloxacin. Repeat titres 2 months later showed Widal test and typhoid IgM were both negative.
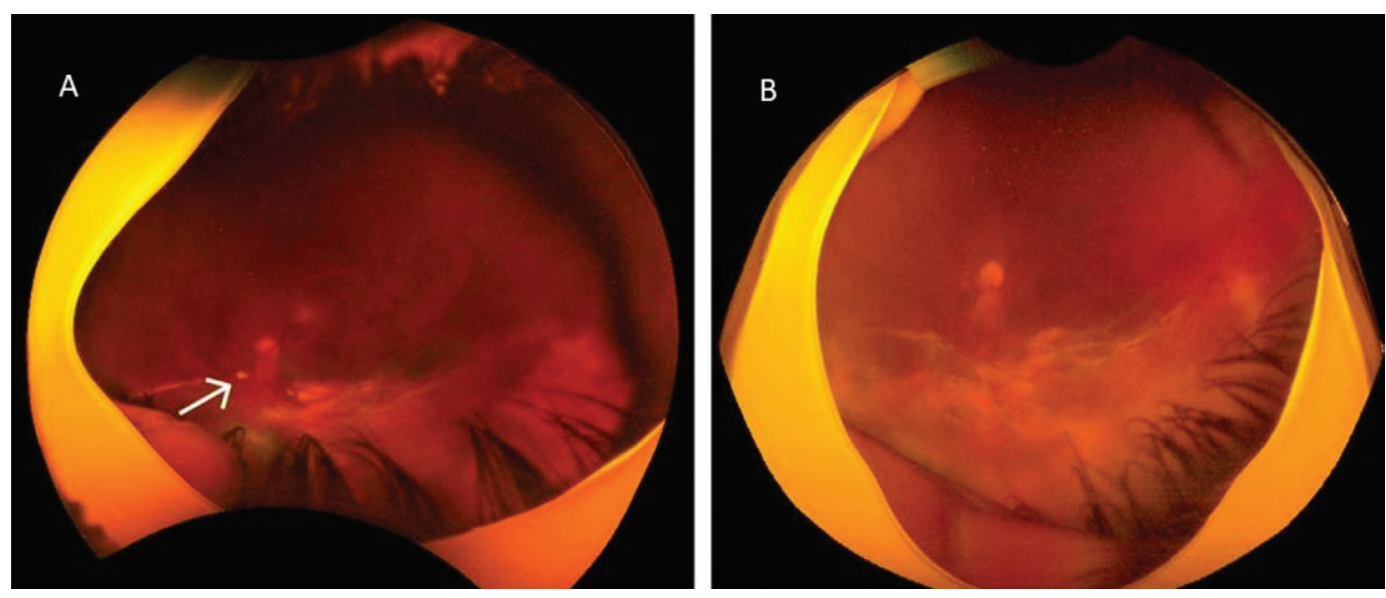

Fig. 1. (A) Wide field fundus photography of the left eye showing retinitis (white arrow), combined retinal detachment with retinal folds inferiorly with sub-retinal exudation with no clinically evident rhegma or hole. Fundus details are not clear due to media opacity because of cataract and vitreous haze in the left eye. (B) Resolved retinitis, with decreased haze resolved exudation with retinal detachment with retinal folds inferiorly with sub-retinal exudation with no rhegma or hole. Post-treatment showed decreased vitreous haze and resolved retinitis. The exudation had reduced with appearance of inferior retinal folds, with thick membrane. 


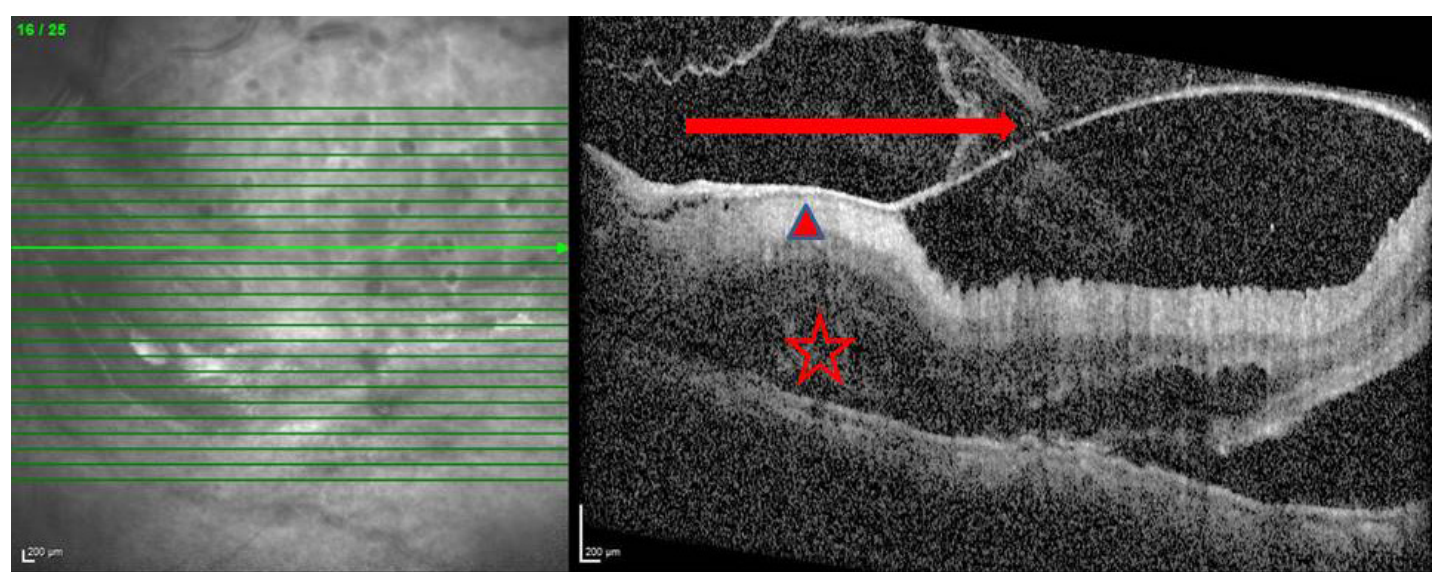

Fig. 2. Optical coherence tomography of the left eye at presentation shows vitreo-macular traction (red arrow), neuro-sensory detachment (red star), distortion of retinal layers (red triangle). The green lines on left are raster scan across the left eye macula and the prominent green line with arrow is the section depicted in the right image.

Five months later at her final visit, her vision was counting fingers 1 metre. Her LE had persistent macular detachment with no obvious breaks. Retinitis lesion had completely resolved (Fig. 1B). OCT of the LE showed reduction in sub-retinal fluid with significant traction with hyper-reflective membrane.

We suggested that she undergo surgery for her retinal detachment involving macula secondary to tractional element and possible hole/tear, which we could not visualise due to bound-down pupil. The patient declined the surgical option.

In 2 Singapore studies published a decade earlier, laboratory reports confirmed cases of enteric fever, the majority of which were typhoid. It was estimated that $75-78 \%$ were imported cases from India, Indonesia and other Southeast Asian countries. The indigenous cases were very few and the results were similar to those in developed countries. ${ }^{2,3}$

In another study of enteric fever involving paediatric age group, $94 \%$ had travelled to typhoid-endemic countries, $70.2 \%$ to the Indian subcontinent, and the rest to Indonesia and Malaysia. All patients infected with multidrug resistant strains had travelled to the Indian subcontinent. ${ }^{4}$

In recent years, immune-mediated retinitis has been reported in association with dengue, chikungunya and rickettsial diseases. ${ }^{5}$ In rare cases, similar manifestations have also been described in patients post-typhoid fever. Post-fever ocular signs described included cotton wool spots, multifocal retinitis, retinal vasculitis, retinal venous occlusion, retinal haemorrhage(s), retinal and optic nerve head oedema, neuroretinitis, large neurosensory detachment, retinal detachment, pseudoretinitis pigmentosa, panophthalmitis, orbital cellulitis and tendonitis. ${ }^{1,5-9}$

Widal test determining "O" and " $\mathrm{H}$ " antigens of Salmonella enterica serotype Typhi and "AH" and "BH" antigens of $S$. enterica serotypes Paratyphi is used for diagnosis. Diagnosis of immune-mediated retinitis is often clinical, based on past history of a febrile illness (4 to 6 weeks prior). It is important to rule out other causes of fever like dengue, chikungunya, rickettsia, West Nile virus, human immunodeficiency virus, toxoplasma, leptospira, tuberculosis, syphilis, connective tissue disorders, systemic lupus erythematosus and rheumatoid arthritis.

A study by Acharya et al. ${ }^{9}$ found that high Widal titres were associated bilateral involvement, extensive lesions and poor visual acuity, all of which were statistically significant.

It is postulated that the direct invasion of the $S$. enterica serotype Typhi or immune-mediated reaction could be attributed to post-infectious immunologic effects. This may lead to an immune response that reacts to self-antigens (for example, heat shock protein and myelin basic protein) or homology between retinal proteins and microbial peptides (similarity between $\mathrm{S}$ antigen and microbial peptides like yeasts, Escherichia coli and hepatitis B virus), or molecular mimicry leading to autoimmunity (S antigen and interphotoreceptor retinoid binding protein). ${ }^{6}$

Our patient had IgM positive for typhoid, indicating an acute infection. Since we were not able to trace the cause of her previous episode of fever, we presumed that this episode was related to typhoid fever. Also the anti-VEGF given to the patient elsewhere before 
presentation to us was possibly due to her macular oedema; unfortunately no records were available.

The patient had both exudations leading to subretinal fluid and tractional element leading to macular detachment. We postulate that the previous anti-VEGF given earlier might have contributed to increased traction that resulted in the clinical sign at presentation to us.

Tractional retinal detachment can occur due to contraction of fibrous tissue. Other presumed mechanisms include the extreme fluctuations in intraocular pressure and deformation of the globe during intravitreal injection, resulting in vitreoretinal traction. ${ }^{10}$

When the patient presented to us, she did not have fever. However, she showed a positive Widal test and IgM for typhoid with ocular inflammation, which needed both antibiotics and oral steroids.

Treatment modalities for post-typhoid ocular manifestations described in the literature include topical non-steroidal anti-inflammatory medications, steroids in various forms including topical, sub-conjunctival, subTenon's, intravenous and oral steroids. ${ }^{3-6}$

Post-treatment visual acuity in one series ranged from $20 / 200$ to $20 / 30$. In most of the cases, the fundus lesions almost resolved, leaving retinal pigment epithelial changes and foveal thinning in cases with severe macular involvement. ${ }^{6}$

Mathur et al. have many decades earlier described post-typhoid retinal detachment associated poor visual outcome, similar to our patient. ${ }^{1}$

Retinal detachment may occur even in the absence of bacteria. Exudative retinal detachment usually responds to oral steroids but may not resolve completely. There may be combined mechanisms of retinal detachment. This rare case illustrates a permanent visual impairment following typhoid fever. Treating physicians should be aware of the ocular complications during typhoid fever.

\section{REFERENCES}

1. Mathur JS, Nema HV, Char JN, et al. Post typhoid retinal detachment. Indian J Ophthalmol 1970;18:135-7.

2. Ty AU, Ang GY, Ang LW, et al. Changing epidemiology of enteric fevers in Singapore. Ann Acad Med Singap 2010;39:889-8.

3. Lee GKM, Tan KW, Goh KT, et al. Trends in importation of communicable diseases into Singapore. Ann Acad Med Singap 2010;39:764-70.

4. Ahmad Hatib NA, Chong CY, Thoon KC, et al. Enteric Fever in a Tertiary Paediatric Hospital: A Retrospective Six-Year Review. Ann Acad Med Singap 2016;45:297-302.

5. Sanjay S, Agrawal S, Mahendradas P, et al. Post fever uveoretinal manifestations in an immunocompetent individual. EMJ Allergy Immunol 2020;5:91-105.

6. Prabhushanker M, Topiwalla TT, Ganesan G, et al. Bilateral retinitis following typhoid fever. Int J Retin Vitr 2017;3:11

7. Hughes EH, Dick AD. The pathology and pathogenesis of retinal vasculitis. Neuropathol Appl Neurobiol 2003;29:325-40.

8. Relhan N, Pathengay A, Albini T, et al. A case of vasculitis, retinitis and macular neurosensory detachment presenting post typhoid fever. Journal of Ophthalmic Inflamm Infect 2014;4:23.

9. Acharya P, Ramamurthy LB, Venugopal KC, et al. Evaluation of posterior segment manifestations following typhoid fever-a clinical study. Ind J Clin Exp Ophthalmol 2018;4:421-5.

10. Ossadon P, Fagan XJ, Lifshitz T, et al. A review of anti VEGF agents for proliferative diabetic retinopathy. Eye (Lond) 2014; 28:510-20.

Srinivasan Sanjay ${ }^{1}$ MRCS (Edin), Ankush $\underline{\text { Kawali }}{ }^{1}{ }_{D N B}(O p h)$, Padmamalini Mahendradas ${ }^{1}{ }_{D N B}(\mathrm{Oph})$,

Naresh Kumar Yadav ${ }^{2}$ FRCS (Glasgow),

Bhujang K $\underline{\text { Shetty }}{ }^{3}$ MS (Oph)

\footnotetext{
${ }^{1}$ Department of Uveitis and Ocular immunology, Narayana Nethralaya, Bengaluru, India

${ }^{2}$ Department of Retina, Narayana Nethralaya, Bengaluru, India

${ }^{3}$ Department of General Ophthalmology and Cataract, Narayana Nethralaya, Bengaluru, India
}

Correspondence: Dr Srinivasan Sanjay, Department of Uveitis and Ocular Immunology, Narayana Nethralaya, 121/C Chord Road, Rajajinagar 1st R block, Bengaluru 560010, Karnataka, India.

Email: sanjaygroup24@gmail.com 